\title{
Shock wave-enhanced emission photoacoustic streaming versus photon-induced photoacoustic streaming modes for clearing root canal bacteria using erbium-doped yttrium aluminum garnet lasers: An in vitro study
}

\section{Xiao-Na Wang \\ Shanxi Provincial People's Hospital \\ Jing Shi ( $\nabla$ crystalshi@163.com ) \\ Shanxi Provincial People's Hospital}

Research article

Keywords: Endodontic treatment, Enterococcus faecalis, Er:YAG laser, photon-induced photoacoustic streaming, root canal, shock wave-enhanced emission photoacoustic streaming

Posted Date: September 22nd, 2019

DOI: https://doi.org/10.21203/rs.2.14714/v1

License: (c) (1) This work is licensed under a Creative Commons Attribution 4.0 International License. Read Full License 


\section{Abstract}

Background : The root canal cleaning efficiency of the photon-induced photoacoustic streaming (PIPS) mode in erbium-doped yttrium aluminum garnet (Er:YAG) lasers is controversial. Shock wave-enhanced emission photoacoustic streaming (SWEEPS), a novel mode in Er:YAG lasers, creates a strong shock wave in a narrow container. We accordingly hypothesized that it may effectively eliminate bacteria from narrow root canals. The present study aimed to compare the bacterial clearance efficiency between the SWEEPS and PIPS modes for Er:YAG lasers designed for root canal treatment.

Methods: The distal and palatal roots of 130 freshly extracted human molars were used. The smear layer was first removed by conventional root canal preparation. All samples were then sterilized in an autoclave. The samples were subsequently exposed to Enterococcus faecalis and incubated for 4 weeks to establish E. faecalis infection models. The models were divided into three groups according to the irrigation method ( $n=40$ per group): $3 \%$ sodium hypochlorite $(\mathrm{NaOCl})$ activated using the SWEEPS Er:YAG laser mode, $3 \% \mathrm{NaOCl}$ activated using the PIPS Er:YAG laser mode, and $3 \% \mathrm{NaOCl}$ without activation.

Results: The bacterial clearance efficiency, based on the colony-forming unit count, was significantly higher in the SWEEPS group than in the PIPS and $\mathrm{NaOCl}$ groups. Moreover, scanning electron microscopy after irrigation revealed that the SWEEPS group had the least number of bacteria.

Conclusions: The SWEEPS mode may be more effective than the PIPS mode for eradicating E. faecalis from root canals.

\section{Background}

Endodontic infection and periapical periodontitis are the most important pathologies involving the dental pulp, with the main cause being bacterial invasion of the root canal system. Therefore, their treatment is based on the elimination of harmful bacteria from the canals [1]. Root canal treatment (RCT) is the most common and effective treatment for these conditions. However, studies [2, 3] have demonstrated a failure rate of $4 \%-15 \%$, even in cases involving uncomplicated treatment. In other words, periapical inflammation may persist or recur after treatment; both these events represent treatment failure. The complex anatomy of the root canal system is the most important reason for failure. Canal enlargement with modern instruments such as stainless steel and nickel-titanium files does not eliminate all microorganisms, particularly those in collateral and apically divergent canals. Therefore, improved cleaning efficiency is essential for a higher RCT success rate [4].

With the development of root canal irrigation technologies, different modalities have become available such as conventional needle irrigation $(\mathrm{Cl})$, manual-dynamic irrigation (MDI), passive ultrasonic irrigation (PUI), and laser-activated irrigation (LAI) [5-7]. Andrabi et al. [8] found that MDI allowed the irrigant solution to easily reach the apical third of the root through effective hydrodynamic activation. This method can also neutralize the vapor lock effect, thus improving the cleaning efficiency. Sluis et al. [9] 
found that PUI was better than $\mathrm{Cl}$ with regard to dentin fragment and planktonic bacteria elimination. The ultrasonic vibration-induced shear force eliminates dentin debris, residual bacteria, pulp tissue, and other infectious substances from the canals [10]. Moreover, the solution effectively enters the main canal and collateral canals, which improves the cleaning performance [11, 12]. However, complete bacterial elimination from the root canal system is not achieved with any of these techniques [13].

The PIPS technology for root canal irrigation was introduced in 2009. Several studies [14-25] have demonstrated its effectiveness. In this method, the laser fiber tip is placed in a pulp cavity filled with the irrigant solution. In this location, it emits pulsed laser light for fluid activation. Absorption of the laser energy superheats the fluid so that its boiling point is reached $[14,16,17,25]$. A vapor bubble consequently forms at the tip. The bubble gradually expands, and then collapses once the maximum value is reached [18]. A series of secondary steam bubbles are triggered during the collapse, and these bubbles also gradually expand and collapse. Under this turbulent sound and light agitation, the irrigant moves throughout the root canal system and flushes out the dentin debris, residual bacteria, pulp tissue, and other infectious substances in the main canal and collateral canals. Thus, the canals are cleaned and disinfected $[19,20,26]$.

Gregorčič et al. [27]found that the bubble collapse generated a powerful shock wave in an infinite container filled with irrigation fluid. This shock wave can eliminate infection from deeper and inaccessible regions. However, another study [16] found that no shock wave was generated in a narrow container. These contrasting findings led researchers to consider measures for improving the cleaning efficiency of the PIPS mode through the generation of a powerful shock wave in narrow areas such as root canals. The shock wave-enhanced emission photoacoustic streaming (SWEEPS) mode, a novel mode for Er:YAG lasers, generates a strong shock wave in a narrow container [28-30]. We therefore hypothesized that Er:YAG laser irrigation using the SWEEPS mode may effectively eliminate bacteria from narrow root canals. Thus, we conducted the present study to compare the bacterial clearance efficiencies of the SWEEPS and PIPS modes for root canal irrigation using Er:YAG lasers.

\section{Methods}

\section{Sample collection and preparation}

The ethics committee of Shanxi Provincial People's Hospital approved the study protocol [Provincial Medical Opinions (2019) No. 3]. Distal and palatal roots of freshly extracted human molars were used for this in vitro study. The selected teeth were untreated and had completely formed apices and root canals. Any calculi and periapical soft tissues were first removed by using an ultrasonic scaler. All samples were then placed in a saline-filled test tube and stored at $4^{\circ} \mathrm{C}$ until use. For the study, the teeth were decoronated using a diamond bur. The standard length of the remaining root was $12 \mathrm{~mm}$. The working length (WL) was set as the standard length minus $1 \mathrm{~mm}$ (i.e., $11 \mathrm{~mm}$ ). K-files (\#10 and \#15; Dentsply Maillefer, Ballaigues, Switzerland) were used to create a glide path for the $\mathrm{WL}$, and ProTaperNext rotary files (Dentsply Sirona, York, PA, USA) were used to shape the canals up to size X3 $(0.3 \mathrm{~mm}, 7 \%)$ according 
to the manufacturer's instructions. After the use of each instrument, the canals were irrigated with $2.5 \mathrm{~mL}$ $3 \%$ sodium hypochlorite $(\mathrm{NaOCl}$ ) solution (Sigma-Aldrich Corporation, St. Louis, MO, USA), which was delivered via a syringe with a $27-G$ side-vented needle. After completing the preparation, the canals were sequentially irrigated with $5 \mathrm{~mL}$ of $17 \%$ ethylenediaminetetraacetic acid (EDTA; Ultradent Products, Inc., South Jordan, UT, USA) and $5 \mathrm{~mL}$ of $3 \% \mathrm{NaOCl}$ for 5 minutes each; this ensured removal of the smear layer. The canals were then flushed for 15 minutes with $20 \mathrm{~mL}$ of $0.9 \%$ physiological saline for the removal of any residual EDTA or $\mathrm{NaOCl}$ solution. The samples were dried at room temperature. To prevent bacterial leakage, we sealed the apical third of all roots with a composite resin and coated the entire root surface with nail polish. Finally, five teeth were randomly selected and subjected to scanning electron microscopy (SEM) (EVO MA10; ZEISS, Oberkochen, Germany) for confirmation of smear layer removal.

The following scoring criteria were used for smear layer removal: 1, no smear layer, $100 \%$ dentinal tubules open; 2 , small amount of scattered smear layer, $80 \%$ dentinal tubules open; 3 , thin smear layer, $60 \%$ dentinal tubules open; 4 , a portion of the root canal wall covered with a thick smear layer; and 5 , root canal wall completely covered by a smear layer. Two experts in the field of dental pulp disease evaluated the SEM images using a double-blind method. A score of 3 or lower met the smear removal standard.

The samples were subsequently placed in glass test tubes filled with $0.9 \%$ physiological saline sterilized at $121^{\circ} \mathrm{C}$ in a 1.5 -Mpa autoclave (LS-150LD; Binjiang Medical Equipment Ltd., Jiangyin, China) for 30 minutes. An inoculating loop was used to collect a loopful of the liquid near the root canal in the test tube. This liquid was inoculated on a sterile plate with blood agar medium and placed in a Tri-Gas incubator (HF-100; Heal Force Bio-meditech Holdings, Ltd., Shanghai, China) for 24 hours. The effects of sterilization were determined by observing the colony growth on the plate.

\section{Establishment ofthe Enterococcus faecalis infection models}

A standard E. faecalis strain (ATCC 29212), which was procured from stocks in the Microbiology Laboratory of Shanxi Provincial People's Hospital, was activated and formulated into a bacterial suspension, the concentration of which was adjusted to 1.0 MCF on an electronic turbidimeter (BioMerieux, Mercy l'Etoile, France). Five tooth samples were placed in a glass tube containing $1 \mathrm{~mL}$ of Enterococcus broth (HB0133-2; Haibo Biotechnology Co., Ltd, Qingdao, China) and $1 \mathrm{~mL}$ of the $E$. faecalis suspension (in total, 25 tubes), which were incubated for 4 weeks at $37^{\circ} \mathrm{C}$ in a Tri-Gas incubator (HF-100; Heal Force). Every 48 hours, the liquid in the tube was changed. At the time of culture solution replacement, $1 \mathrm{~mL}$ of liquid near the root canal was collected and incubated for 24 hours on a plate containing blood agar medium. The presence of other bacteria was ruled out by analyzing the formed colonies using a fully automated rapid mass spectrometry detection system (Microflex LT/SH; Bruker Daltonik, Bremen, Germany). After 4 weeks, in vitro E. faecalis infection models were successfully established. To confirm E. faecalis colonization, five samples were randomly selected and observed by using SEM. 


\section{Bacterial sampling and counting before irrigation}

In a biosafety cabinet (HFSafe 1200; Heal Force Bio-meditech Holdings, Ltd.), the culture solution in the canals was carefully blotted by using sterile paper tips. The canals were then rinsed with $1 \mathrm{~mL}$ of $0.9 \%$ sterile saline to flush out unattached bacteria. Thereafter, three sterile paper tips saturated with $0.9 \%$ saline were successively inserted up to the WL and repeatedly rubbed against the inner canal walls. After 1 minute, the paper tips were placed in $1 \mathrm{~mL}$ of $0.9 \%$ sterile physiological saline and shaken on a vortex mixer (XW-80A; Jingke Industrial Co., Ltd., Shanghai, China) for 5 minutes for deployment of the bacterial suspension. This suspension was serially diluted with physiological saline (up to $10^{-6}$ ), with a volume ratio of $1: 10$. To count the bacteria, $0.1-\mathrm{mL}$ aliquots containing appropriate dilutions of each sample were spread onto blood agar plates and incubated for 24 hours at $37^{\circ} \mathrm{C}$ in the Tri-Gas incubator (HF-100; Heal Force Bio-meditech Holdings, Ltd.). The colony-forming unit (CFU) number in the entire plate was then counted and recorded.

\section{Irrigation protocols}

We randomly divided the 120 roots into two experimental groups and one control group $(n=40$ each) according to the irrigation protocol: $3 \% \mathrm{NaOCl}$ activation with an Er:YAG laser (LightWalkers ATS; Fotona, Ljubljana, Slovenia) using the SWEEPS mode for 60 seconds (SWEEPS group); $3 \% \mathrm{NaOCl}$ activation with an Er:YAG laser (LightWalkers ATS) using the PIPS mode for 60 seconds (PIPS group), and $3 \% \mathrm{NaOCl}$ irrigation without activation for 60 seconds (control group).

The parameters for the SWEEPS and PIPS modes are presented in Table 1. In the SWEEPS group, the canals were subjected to three cycles involving $3 \% \mathrm{NaOCl}(2 \mathrm{~mL}$ each) activation using the SWEEPS mode for 20 seconds, $0.9 \%$ sterile physiological saline irrigation $(2 \mathrm{~mL}$ each) for 20 seconds, and a rest interval of 20 seconds. The same regimen was followed in the PIPS group, where $\mathrm{NaOCl}$ was activated using the PIPS mode. In the control group, the canals were subjected to three 20 -second cycles of $3 \%$ $\mathrm{NaOCl}$ ( $2 \mathrm{~mL}$ each) irrigation via a syringe with a $27-\mathrm{G}$ side-vented needle without any activation, $0.9 \%$ sterile physiological saline irrigation ( $2 \mathrm{~mL}$ each) for 20 seconds, and a rest period of 20 seconds.

Table 1. Parameters for Er:YAG laser irrigation of root canals using the shock wave-enhanced emission photoacoustic streaming mode and the photon-induced photoacoustic streaming mode

\begin{tabular}{llllll}
\hline Mode & Pulse $(\mathrm{mJ})$ & Frequency $(\mathrm{Hz})$ & Power $(\mathrm{W})$ & $\lambda(\mu \mathrm{m})$ & Water and air \\
\hline SWEEPS & 20 & 15 & 0.3 & 2.94 & Turn off \\
PIPS & 20 & 15 & 0.3 & 2.94 & Turn off \\
\hline
\end{tabular}

Er:YAG: erbium-doped yttrium aluminum garnet, PIPS: photon-induced photoacoustic streaming, SWEEPS: shock wave-enhanced emission photoacoustic streaming 


\section{Bacterial sampling and counting after irrigation}

The method used for bacterial sampling and counting after irrigation was the same as that used before irrigation. On the basis of the obtained values, we calculated the bacterial clearance rate by using the following formula: bacterial clearance rate $(\%)=(E-F) / E \times 100$. In this paper, $E$ and $F$ represent the number of bacterial colonies before and after irrigation, respectively. Based on the bacterial clearance rate, the bacterial clearance efficiency of each method was evaluated. For more intuitive demonstration of the bacterial clearance efficiency, the number of remaining bacteria in the irrigated samples from the three groups was observed by SEM.

\section{Statistical analysis}

All data were statistically analyzed using SPSS, version 22.0 (IBM, Chicago, IL, USA). We used the Kruskal-Wallis test to detect statistically significant differences between groups. Intergroup comparisons were conducted using nonparametric one-way analysis of variance. The bacterial clearance rates are presented as medians and interquartile ranges. A $p$-value of $\leq 0.05$ was considered statistically significant.

\section{Results}

\section{Scanning electron microscopy images before irrigation}

Figure 1 shows an SEM image of a middle canal after smear layer removal. Figure 2 shows SEM images of a successfully established $E$. faecalis infection model.

\section{Scanning electron microscopy images after irrigation}

The SEM images for the SWEEPS group showed nearly complete $E$. faecalis elimination from the canals (Fig. 3a), whereas the SEM images for the PIPS group showed some residual bacteria (Fig. 3b). In the control group, a large number of residual bacteria were visible (Fig. 3c). Overall, the SEM images indicated a qualitative decrease in the bacterial counts in the SWEEPS and PIPS groups relative to the counts in the control group (Fig. 3).

The bacterial clearance rates calculated from the CFU counts before and after irrigation are shown in Table 2. All irrigation regimens significantly reduced bacterial growth $(p<0.001)$. The bacterial clearance rates of $88.6 \%, 58.5 \%$, and $34.4 \%$ in the SWEEPS, PIPS, and control groups, respectively, were significantly different $(p<0.05)$.

Table 2. Statistical analysis of colony-forming units for the evaluation of bacterial clearance in different root canal irrigation groups 


\begin{tabular}{lllllll}
\hline & No. (median) & $\mathrm{Q}\left(\mathrm{P}_{25}-\mathrm{P}_{75}\right)$ & \multicolumn{2}{c}{ Range } & \multirow{2}{*}{$\mathrm{c}^{2}$} & $p$ \\
\cline { 4 - 5 } & & & $\mathrm{Min}$ & \multicolumn{2}{c}{$\operatorname{Max}$} \\
\hline SWEEPS & 40 & $100.00 \% *, * *$ & $97.94 \%-100 \%$ & $42.86 \%-100 \%$ & & \\
PIPS & 40 & $85.56 \% *$ & $64.59 \%-99.84 \%$ & $0.00 \%-100 \%$ & 50.14 & $<0.001$ \\
Control & 40 & $64.34 \%$ & $0.00 \%-79.43 \%$ & $0.00 \%-100 \%$ & & \\
\hline
\end{tabular}

Er:YAG: erbium-doped yttrium aluminum garnet, PIPS: photon-induced photoacoustic streaming, SWEEPS: shock wave-enhanced emission photoacoustic streaming

For SWEEPS, 3\% sodium hypochlorite ( $\mathrm{NaOCl}$ ) was activated with the Er:YAG laser in the shock wave-enhanced emission photoacoustic streaming mode. For PIPS, 3\% $\mathrm{NaOCl}$ was activated with the Er:YAG laser in the photoninduced photoacoustic streaming mode. For Control, 3\% $\mathrm{NaOCl}$ was not activated.

* Indicates a significant difference from the value for group control $(p<0.05)$.

** Indicates a significant difference from the value for group PIPS $(p<0.05)$.

\section{Discussion}

In the present study using the E. faecalis infection model, we hypothesized that Er:YAG laser irrigation using the SWEEPS mode may effectively eliminate bacteria from narrow root canals. Our comparison of the bacterial clearance efficiency between the SWEEPS and PIPS modes for root canal irrigation using Er:YAG lasers revealed that the SWEEPS mode resulted in near-complete elimination of $E$. faecalis from root canals via enhanced pressure waves travelling at shock speeds. Previous studies [31, 32] have demonstrated high detection rates for $E$. faecalis in root canals with secondary infection. Owing to its special properties, E. faecalis forms a single bacterial biofilm on root canal surfaces. Therefore, an $E$. faecalis infection model is often used as a standard model for evaluation of the cleaning ability of irrigation protocols

In the SWEEPS mode, a pair of individual laser pulses is emitted to accelerate the effects of fluid cavitation dynamics. An initial laser pulse is emitted at time $T_{0}$, and the initial vapor bubble and smaller secondary vapor bubbles are simultaneously formed at the fiber tip. These bubbles gradually expand until they reach the maximum volume at time $T_{\max }$. The bubble volume then gradually decreases. During their collapse, subsequent laser pulses are delivered to the solution at time $T_{\text {opt }}$. At that point, several secondary bubbles are triggered. The pressure waves created by these bubbles induce the violent collapse of the initial and secondary bubbles, and thereby accelerate fluid motion. Powerful shock waves are eventually generated throughout the root canal, which significantly improves clearance efficiency [20, 
33]. In the present study, this efficiency was proven by the bacterial clearance rates and SEM images, which showed that the SWEEPS mode caused near-complete elimination of E. faecalis from the observed canals.

Notably, shock waves cannot be increased in narrow spaces such as root canals simply by increasing the energy of a single Er:YAG laser pulse [20]. An increase in the energy of a single pulse will enlarge cavitation bubbles and slow bubble oscillation dynamics at higher laser energies. The optimal shaping of the laser pulse emission temporally by using the SWEEPS technique enables amplification of the photoacoustic streaming effects [34].

We believe that the SWEEPS mode will improve the root canal cleaning efficiency and aid in direct smear layer removal. One limitation of this study was that we did not study the ability of the SWEEPS mode to remove the smear layer because of time constraints and other factors. Future studies are necessary to clarify this aspect.

\section{Conclusion}

When using Er:YAG laser irrigation, the SWEEPS mode may be superior to the PIPS mode in terms of bacterial clearance from infected root canals. The outcomes of root canal treatment can be negatively affected if residual bacteria grow and cause problems such as inflammation. In this scenario, the patient would require repeat treatment, which can be prevented by more thorough cleansing of the infected canal(s). Our findings regarding the efficacy of the SWEEPS mode for Er:YAG laser irrigation could potentially improve the outcomes of root canal treatment and prevent the need for re-treatment. In addition, they could form a basis for the development of new strategies for root canal irrigation in clinical practice. We hope to monitor the patients treated with the SWEEPS mode in order to evaluate its longterm effectiveness.

\section{Abbreviations}

CFU, colony-forming unit

$\mathrm{Cl}$, conventional needle irrigation

EDTA, ethylenediaminetetraacetic acid

Er:YAG, erbium-doped yttrium aluminum garnet

LAl, laser-activated irrigation

MDI, manual-dynamic irrigation

$\mathrm{NaOCl}$, sodium hypochlorite 
PIPS, photon-induced photoacoustic streaming

PUl, passive ultrasonic irrigation

$\mathrm{RCT}$, root canal treatment

SEM, scanning electron microscopy

SWEEPS, shock wave-enhanced emission photoacoustic streaming

WL, working length.

\section{Declarations}

Ethical approval and consent to participate: The ethics committee of Shanxi Provincial People's Hospital approved the study protocol [Provincial Medical Opinions (2019) No. 3]. The need for informed consent was not applicable for this type of study.

Consent for publication: Not applicable.

Availability of data and materials: The datasets used and/or analysed during the current study are available from the corresponding author on reasonable request.

Competing interests: The authors declare that they have no competing interests.

Funding: The study was funded by the application and promotion project fund of laser technology in the field of stomatology of Shanxi Provincial Department of Science and Technology (grant number 201704D131027). The sponsor had no role in the study design; in the collection, analysis, and interpretation of data; in the writing of the report; and in the decision to submit the article for publication.

Authors' contributions: XNW: experimental operation, data analysis, statistical analysis, article writing and modification; JS: project design and guidance, article writing and modification. All authors read and approved the final manuscript.

Acknowledgements: We wish to thank Dr. Yang Xiaowen, Dr. Yang Zhining, and Dr. Zhang Yanjun for their support during the study.

\section{References}

1. Huang $D$, Tan $X$, Zhang $L$, Zhou $X$. Confusion and solution for root canal working length determination. Hua Xi Kou Qiang Yi Xue Za Zhi. 2016;34:109-14

2. Smith CS, Setchell DJ, Harty FJ. Factors influencing the success of conventional root canal therapyA five-year retrospective study. Int Endod J. 1993;26:321-33 
3. Siqueira JF Jr, Alves FR, Almeida BM, de Oliveira JC, Rôças IN. Ability of chemomechanical preparation with either rotary instruments or self-adjusting file to disinfect oval-shaped root canals. $J$ Endod. 2010;36:1860-5

4. van der Sluis LW. [Endodontics in motion: new concepts, materials and techniques 3. The role of irrigants during root canal treatment]. Ned Tijdschr Tandheelkd. 2015;122:533-8. Article in Dutch.

5. Deleu E, Meire MA, De Moor RJ. Efficacy of laser-based irrigant activation methods in removing debris from simulated root canal irregularities. Lasers Med Sci. 2015;30:831-5.

6. Zehnder M. Root canal irrigants. J Endod. 2006;32:389-98.

7. Williamson AE, Sandor AJ, Justman BC. A comparison of three nickel titanium rotary systems, Endo Sequence, ProTaper universal, and profile GT, for canal-cleaning ability. J Endod. 2009;35:107-9.

8. Andrabi SM, Kumar A, Mishra SK, Tewari RK, Alam S, Siddiqui S. Effect of manual dynamic activation on smear layer removal efficacy of ethylenediaminetetraacetic acid and SmearClear: an in vitro scanning electron microscopic study. Aust Endod J. 2013;39:131-6.

9. van der Sluis LW, Versluis M, Wu MK, Wesselink PR. Passive ultrasonic irrigation of the root canal: a review of the literature. Int Endod J. 2007;40:415-26.

10. Rodríguez-Figueroa C, McClanahan SB, Bowles WR. Spectrophotometric determination of irrigant extrusion using passive ultrasonic irrigation, EndoActivator, or syringe irrigation. J Endod. 2014;40:1622-6.

11. Yuanyuan C, Wenhui Z, Bin G, Xiaolong G, Shilu H, Hu L, et. al. [Cleaning effect of ultrasonic activation as an adjunct to syringe irrigation of root canals: a systematic review]. Hua Xi Kou Qiang Yi Xue Za Zhi. 2015;33:145-52. Article in Chinese.

12. Andrabi SM, Kumar A, Zia A, Iftekhar $\mathrm{H}$, Alam S, Siddiqui S. Effect of passive ultrasonic irrigation and manual dynamic irrigation on smear layer removal from root canals in a closed apex in vitro model. J Investig Clin Dent. 2014;5:188-93.

13. Jiang LM, Lak B, Eijsvogels LM, Wesselink P, van der Sluis LW. Comparison of the cleaning efficacy of different final irrigation techniques. J Endod. 2012;38:838-41.

14. Blanken J, De Moor RJ, Meire M, Verdaasdonk R. Laser induced explosive vapor and cavitation resulting in effective irrigation of the root canal. Part 1: a visualization study. Lasers Surg Med. 2009;41:514-9.

15. De Moor RJ, Blanken J, Meire M, Verdaasdonk R. Laser induced explosive vapor and cavitation resulting in effective irrigation of the root canal. Part 2: evaluation of the efficacy. Lasers Surg Med. 2009;41:520-3.

16. Lukač N, Gregorčič P, Jezeršek M. Optodynamic phenomena during laser-activated irrigation within root canals. Int J Thermophys. 2016;37:1-8.

17. Lukac N, Zadravec J, Gregorcic P, Lukac M, Jezeršek M. Wavelength dependence of photon-induced photo-acoustic streaming technique for root canal irrigation. J Biomed Opt. 2016;21:75007. 
18. Kuhn K, Rudolph H, Luthardt RG, Stock K, Diebolder R, Hibst R. Er:YAG laser activation of sodium hypochlorite for root canal soft tissue dissolution. Lasers Surg Med. 2013;45:339-44.

19. Koch JD, Jaramillo DE, DiVito E, Peters OA. Irrigant flow during photon-induced photoacoustic streaming (PIPS) using Particle Image Velocimetry (PIV). Clin Oral Investig. 2016;20:381-6.

20. Arslan H, Akcay M, Ertas H, Capar ID, Saygili G, Meşe M. Effect of PIPS technique at different power settings on irrigating solution extrusion. Lasers Med Sci. 2015;30:1641-5.

21. Divito E, Peters OA, Olivi G. Effectiveness of the erbium:YAG laser and new design radial and stripped tips in removing the smear layer after root canal instrumentation. Lasers Med Sci. 2012;27:273-80.

22. Peeters HH, Suardita K. Efficacy of smear layer removal at the root tip by using ethylenediaminetetraacetic acid and erbium, chromium: yttrium, scandium, gallium garnet laser. $J$ Endod. 2011;37:1585-9.

23. Akcay M, Arslan H, Durmus N, Mese M, Capar ID. Dentinal tubule penetration of AH Plus, iRoot SP, MTA fillapex, and guttaflow bioseal root canal sealers after different final irrigation procedures: a confocal microscopic study. Lasers Surg Med. 2016;48:70-6.

24. Mir M, Gutknecht N, Poprawe R, Vanweersch L, Lampert F. Visualising the procedures in the influence of water on the ablation of dental hard tissue with erbium: yttrium-aluminium-garnet and erbium, chromium: yttrium-scandium-gallium-garnet laser pulses. Lasers Med Sci. 2009;24:365-74.

25. Peters OA, Bardsley S, Fong J, Pandher G, Divito E. Disinfection of root canals with photon-initiated photoacoustic streaming. J Endod. 2011;37:1008-12.

26. Lloyd A, Uhles JP, Clement DJ, Garcia-Godoy F. Elimination of intracanal tissue and debris through a novel laser-activated system assessed using high-resolution micro-computed tomography: a pilot study. J Endod. 2014;40:584-7.

27. Gregorčič P, Jezeršek M, Mozina J. Optodynamic energy-conversion efficiency during an Er:YAG-laser pulse delivery into a liquid through different fiber-tip geometries. J Biomed Opt. 2012;17:075006.

28. de Icaza-Herrera M, Fernández F, Loske AM. Combined short and long-delay tandem shock waves to improve shock wave lithotripsy according to the Gilmore-Akulichev theory. Ultrasonics. 2015;58:539.

29. Gregorčič P, Možina J, Močnik G. Measurements of cavitation bubble dynamics based on a beamdeflection probe. Appl Phys A. 2008;93:901-5.

30. Loske AM, Prieto FE, Fernandez F, van Cauwelaert J. Tandem shock wave cavitation enhancement for extracorporeal lithotripsy. Phys Med Biol. 2002;47:3945-57.

31. Tennert C, Fuhrmann M, Wittmer A, Karygianni L, Altenburger MJ, Pelz K, et. al. New bacterial composition in primary and persistent/secondary endodontic infections with respect to clinical and radiographic findings. J Endod. 2014;40:670-7.

32. Zhang C, Du J, Peng Z. Correlation between Enterococcus faecalis and persistent intraradicular infection compared with primary intraradicular infection: a systematic review. J Endod. 2015;41:1207-13. 
33. Lukac N, Muc BT, Jezersek M, Lukac M. Photoacoustic endodontics using the novel SWEEPS Er:YAG laser modality. Laser Health Acad. 2017;1:1-7.

34. Chen X, Xu RQ, Chen JP, Shen ZH, Jian L, Ni XW. Shock-wave propagation and cavitation bubble oscillation by Nd: YAG laser ablation of a metal in water. Appl Opt. 2004;43:3251-7

\section{Figures}

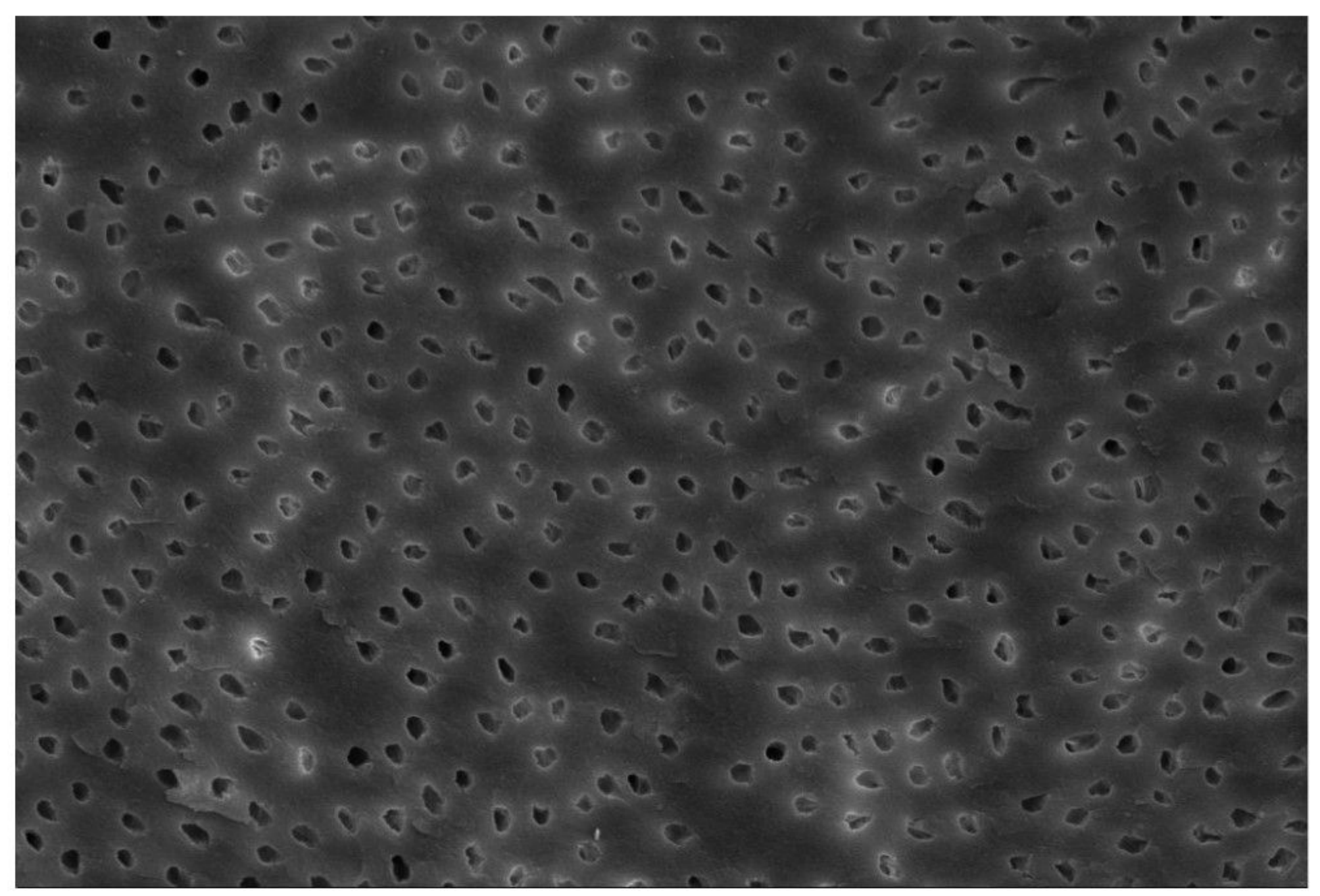

\section{Figure 1}

A scanning electron microscopy image shows elimination of the smear layer in the middle canal. $(\times 1000$ magnification) 
a

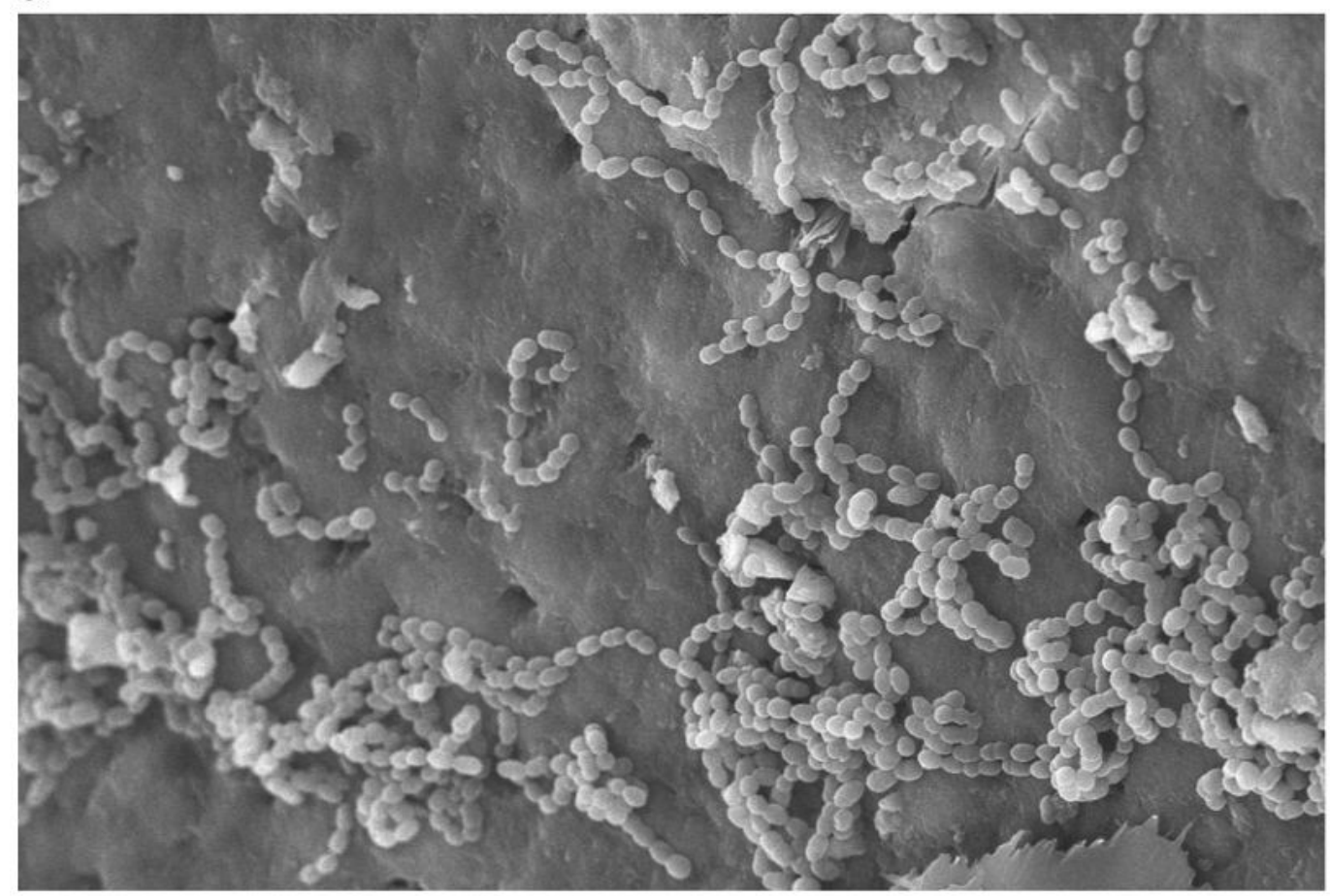

\section{b}

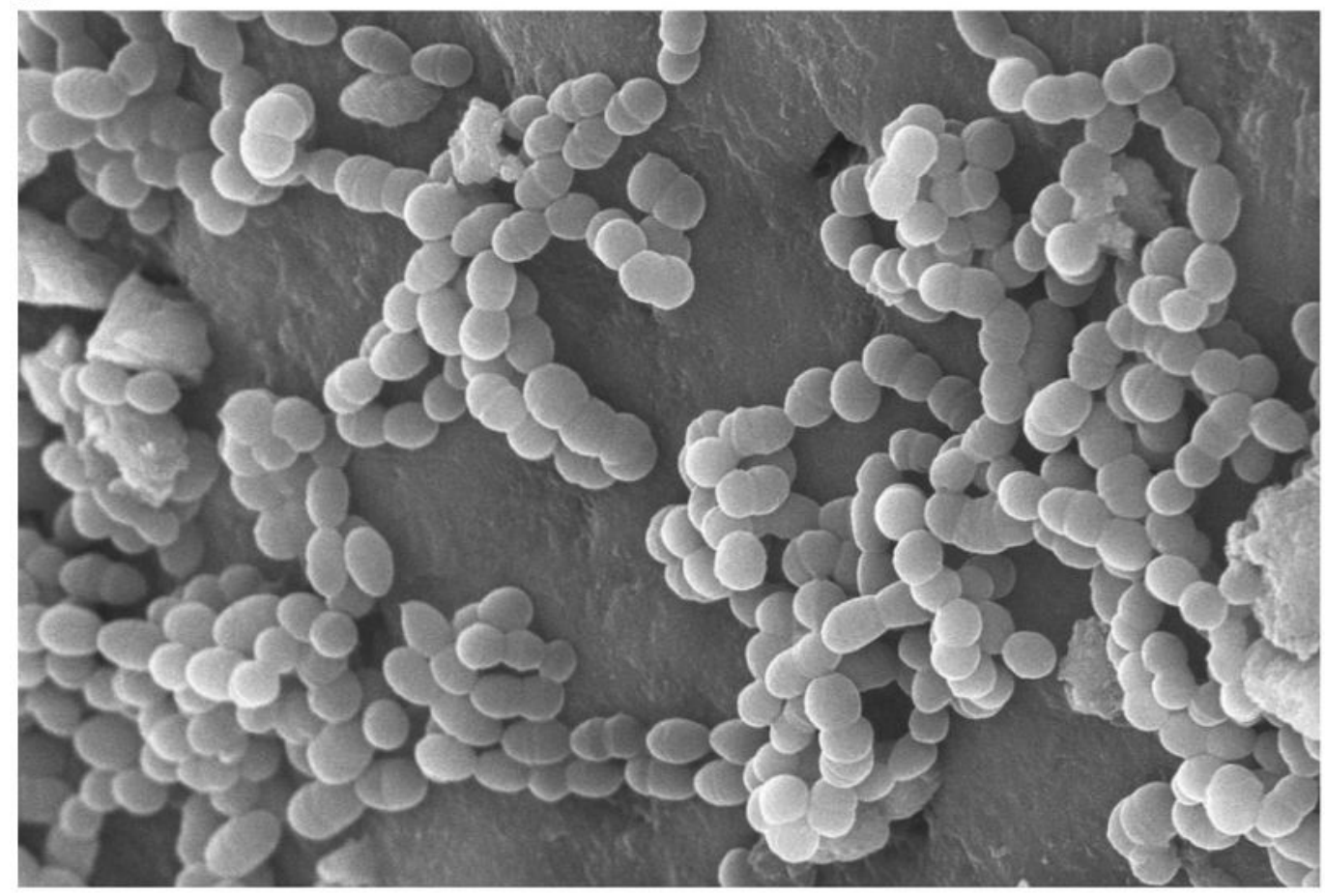

\section{Figure 2}

Scanning electron microscopy images show a successfully established Enterococcus faecalis biofilm at $a \times 2000$ magnification and $b \times 5000$ magnification 

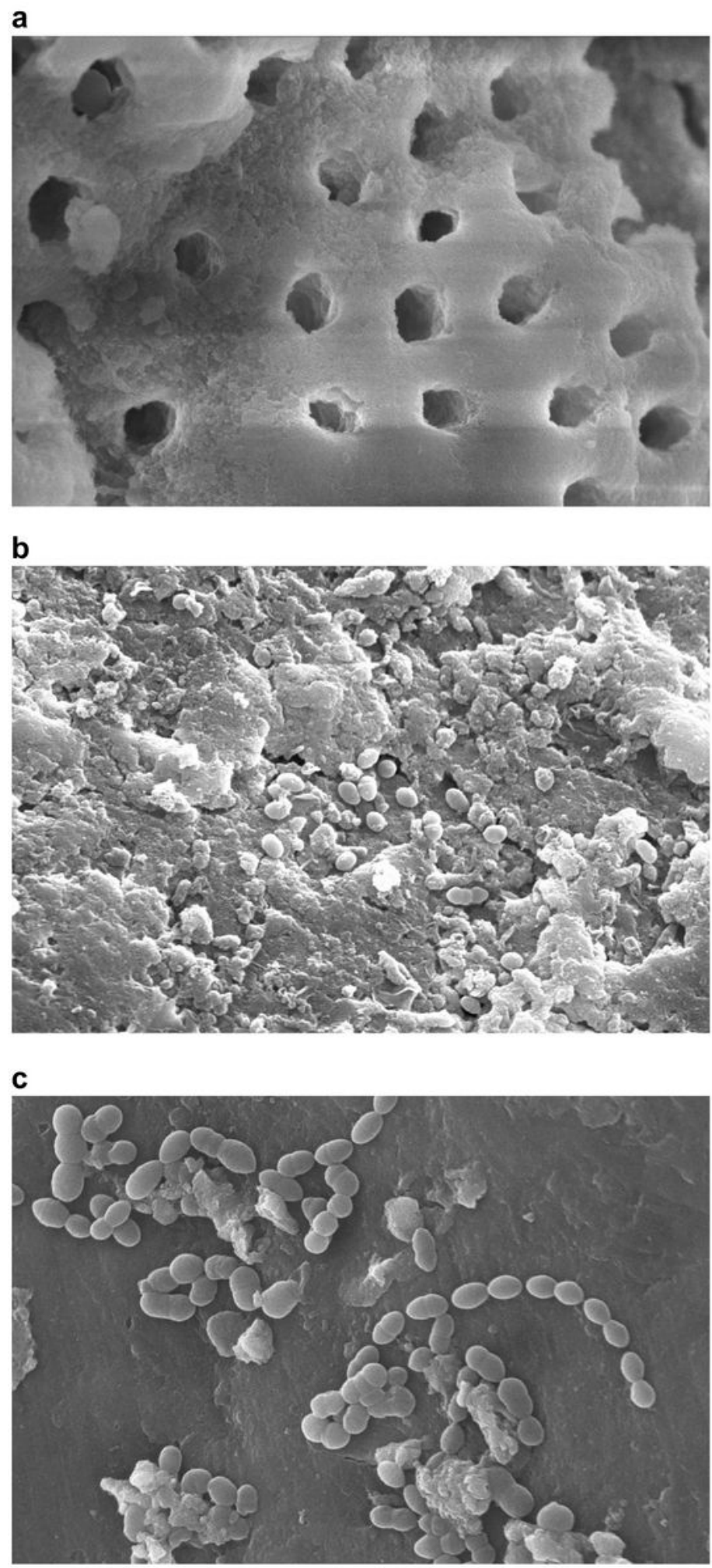

\section{Figure 3}

Scanning electron microscopy images of the middle root canals show the bacterial clearance effects with three mode. a Er:YAG laser activation using the SWEEPS mode ( $\times 5000$ magnification) The Enterococcus faecalis biofilm is almost completely removed. $b$ Er:YAG laser activation using the PIPS mode $(\times 5000$ magnification) Some residual bacteria are present in the root canal. c No laser activation $(\times 5000$ magnification) A large number of bacteria can be seen in the root canal 